\title{
Spontaneous Fungal Ascites Infection in Patients with Cirrhosis: An Analysis of 10 Cases
}

\author{
Beiling Li · Chao Yang · Zhiping Qian · Yan Huang · Xianbo Wang • \\ Guotao Zhong · Jinjun Chen
}

Received: November 22, 2020 / Accepted: February 20, 2021 / Published online: March 11, 2021

(C) The Author(s) 2021

\begin{abstract}
Introduction: Spontaneous fungal ascites infection is a rare but devastating complication of cirrhosis. We aimed to analyse the clinical features, short-term mortality, and treatment of spontaneous fungal ascites infection in patients with cirrhosis.

Methods: We retrospectively studied ten patients with cirrhosis and spontaneous fungal ascites infections, and the clinical characteristics and outcomes were obtained.
\end{abstract}

Beiling Li and Chao Yang contributed equally and share first authorship.

B. Li · G. Zhong · J. Chen $(\bowtie)$

Hepatology Unit, Department of Infectious

Diseases, Nanfang Hospital, Southern Medical

University, Guangzhou, China

e-mail: chjj@smu.edu.cn

\section{Yang}

Department of Laboratory Medicine, Nanfang

Hospital, Southern Medical University, Guangzhou, China

Z. Qian

Department of Liver Intensive Care Unit, Shanghai Public Health Clinical Centre, Fudan University, Shanghai, China

\section{Y. Huang}

Department of Infectious Diseases, Hunan Key Laboratory of Viral Hepatitis, Xiangya Hospital, Central South University, Hunan, China
Result: The patients' mean age was $64 \pm 13$ years, and seven of the ten patients were men. Cirrhosis was primarily caused by infection with the hepatitis B virus. Candida albicans was the most frequently isolated fungus isolated from the ascites fluid. Three of the ten patients fulfilled the criteria of acute-on-chronic liver failure (ACLF) at baseline, and three of the remaining seven patients developed ACLF during hospitalisation. Of the ten patients, six had acute kidney injury (AKI), and six died within 28 days. Three patients did not receive antifungal treatment during hospitalisation because they died undiagnosed because of delays in the reporting of laboratory results.

\author{
X. Wang \\ Center of Integrative Medicine, Beijing Ditan \\ Hospital, Capital Medical University, Beijing, China \\ J. Chen \\ Hepatology Unit, Zengcheng Branch, Nanfang \\ Hospital, Southern Medical University, Guangzhou, \\ China \\ Z. Qian · Y. Huang · X. Wang · J. Chen \\ Chinese (acute on) Chronic Liver Failure \\ Consortium (Ch-CLIF.C), Shanghai, China
}


Conclusion: Patients with spontaneous fungal ascites infection had high incidence of AKI and 28-day mortality. Fungal cultures of ascitic fluid from patients with cirrhosis should be recommended to ensure optimal clinical management, especially in patients with severe liver disease and who received inadequate empirical antibacterial therapy. Hence, future studies should focus on the early diagnosis of fungal infection in patients with cirrhosis.

Keywords: Cirrhosis; Fungiascites; Spontaneous fungal peritonitis; Treatment

\section{Key Summary Points}

\section{Why carry out this study?}

Spontaneous fungal ascites infection is a rare but devastating complication in patients with cirrhosis.

Few studies have been reported on this topic.

\section{What was learned from the study?}

Patients with spontaneous fungal ascites infection had high incidence of AKI and 28-day mortality.

Fungal cultures of ascitic fluid from patients with cirrhosis should be performed to ensure optimal clinical management, especially from those with critical liver disease and poorly administered empirical antimicrobial therapy.

The issue of delayed diagnosis is prominent and new technology for early pathogen detection is necessary.

\section{DIGITAL FEATURES}

This article is published with digital features, including a summary slide, to facilitate understanding of the article. To view digital features for this article go to https://doi.org/10.6084/ m9.figshare.14054375.

\section{INTRODUCTION}

Patients with cirrhosis are at risk of developing various types of infection and have a fourfold higher risk of mortality [1]. Ascites infection is a common complication in patients with cirrhosis. According to the type of pathogen, patients with cirrhosis may develop two types of spontaneous peritonitis: spontaneous bacterial peritonitis (SBP) and spontaneous fungal peritonitis (SFP). SBP is the most common type of infection and has been clearly described in international guidelines [2, 3]. SFP is a rare, less-recognised complication of cirrhosis, with an incidence of less than 5\% [4]. Furthermore, fungiascites is another form of spontaneous fungal ascites infection in patients with liver cirrhosis, which is characterised by a positive fungal ascitic fluid culture, but with an ascites neutrophil cell count less than 250 cells $/ \mathrm{mm}^{3}$.

Fungal infections often occur in hospitalised patients with cirrhosis and critical liver disorders, especially those with acute-on-chronic liver failure (ACLF) $[5,6]$. Patients with endstage liver disease, such as invasive pulmonary aspergillosis, who developed common fungal infections are at higher risk of short-term mortality (73.4-100\%) [7-10]. For patients with refractory ascites, the ascitic fluid contains agents with a low immune activity, providing a good environment for pathogen growth, including bacterial and fungus [11]. This can result in ascitic fluid infection and other related complications, especially acute kidney injury (AKI) as well as progression of ACLF in decompensated patients with cirrhosis. Bucsics et al. reported that 14 patients with cirrhosis and spontaneous fungal ascites infection had a poor prognosis [12]. Only a few studies have investigated patients with SFP, and there are no established guidelines regarding the management of fungal infections in patients with cirrhosis. Thus, this study aimed to observe the clinical features and prognosis of spontaneous fungal ascites infection in ten patients. 


\section{METHODS}

We retrospectively studied ten patients with cirrhosis and spontaneous fungal ascites infections who were hospitalised during 2013-2017 in Nanfang Hospital, Shanghai Public Health Clinical Centre, Xiangya Hospital, and Ditan Hospital. Diagnostic paracentesis was performed, and ascitic fluid was inoculated in the blood culture bottles at the bedside of patients with cirrhosis. The demographic data, results of routine laboratory tests, findings of microbiological analyses, and treatment regimens were recorded. The fungus was isolated from ascitic fluid cultures. Identification and susceptibility tests of the isolated fungus were performed using the Vitek 2 automated system (bioMerieux, Craponne, France). Drug sensitivity testing was performed according to the guidelines of the Clinical and Laboratory Standard Institute [13]. The antimicrobial susceptibility test results were available within $48-72 \mathrm{~h}$ after the ascitic fluid culture indicated positive results.

Cirrhosis was diagnosed on the basis of the patients' clinical manifestations, radiological findings, and/or histological findings. The criteria for diagnosing SFP were as follows: ascites neutrophil cell count greater than 250 cells/ $\mathrm{mm}^{3}$ and isolation of fungi in ascitic fluid cultures. Fungiascites was defined as an ascites neutrophil cell count less than 250 cells $/ \mathrm{mm}^{3}$ and isolation of fungi from the ascitic fluid. AKI and ACLF were defined according to the criteria established by the International Club of Ascites (ICA) [14] and the European Association for the Study of Liver Failure (EASL-CLIF) [15], respectively. Patients with fungal ascites infection were diagnosed with sepsis if they fulfilled at least two of the following criteria: body temperature less than $36^{\circ} \mathrm{C}$ or greater than $38^{\circ} \mathrm{C}$, heart rate greater than 90 beats per minute, respiratory rate greater than $20 / \mathrm{min}$, white blood cells less than $4000 / \mu \mathrm{L}$ or greater than $12,000 / \mu \mathrm{L}$, or immature neutrophils greater than 10\%. Evaluations regarding the clinical efficiency of antibiotic therapy were entrusted to the attending physicians and were based on the clinical, laboratory, and microbiological analysis results [16].
Quantitative variables are expressed as mean \pm standard deviation or median (interquartile range), whereas categorical variables are expressed as percentages. IBM SPSS Statistics 21.0 (IBM Corp., Armonk, NY, USA) was used to perform all statistical analyses.

The study received ethics approval from the ethics committee of all the participating medical centres (Nanfang Hospital, Shanghai Public Health Clinical Centre, Xiangya Hospital and Beijing Ditan Hospital). The ethics committees waived the informed consent because the data were de-identified and individual patient's data were not published. The study was performed in accordance with the declaration of Helsinki 1964 and its later amendments.

\section{RESULTS}

\section{Patients' Characteristics}

We retrospectively studied ten patients with cirrhosis and spontaneous fungal ascites infections from four hospitals. The baseline characteristics of the ten patients are presented in Table 1 . Hepatitis B virus (8/10) was the primary agent causing cirrhosis. The patients' mean age was $64 \pm 13$ years, and seven of the ten patients were men. Eight patients were admitted because of a larger volume of ascites, whereas two had new-onset ascites during hospitalisation. The median time interval between ascites diagnosis and paracentesis was 2.5 days (range 1.8-4.2). Candida albicans was the most commonly isolated fungus in this study. The mean model for end-stage liver disease (MELD) score among the ten patients was $22 \pm 8$. Three patients $(30 \%)$ who fulfilled the EASL-CLIF criteria were diagnosed with ACLF. Two patients were diagnosed with sepsis at the time of ascites paracentesis. Of the ten patients, six patients developed AKI. For seven patients with acute decompensation who did not fulfil the EASL-CLIF criteria for diagnosing ACLF, three were developed ACLF during hospitalisation. Six patients died within 28 days. In this study, four (40\%) patients with cirrhosis were diagnosed with fungiascites, whereas six $(60 \%)$ were diagnosed with SFP. The 
Table 1 Baseline characteristics of the patients

\begin{tabular}{|c|c|}
\hline & Patients included $(n=10)$ \\
\hline Male, $n(\%)$ & $7(70)$ \\
\hline Age (years), mean (SD) & $64(13)$ \\
\hline \multicolumn{2}{|l|}{ Etiology of cirrhosis, $n$ (\%) } \\
\hline $\mathrm{HBV}$ & $6(60)$ \\
\hline HBV plus alcohol & $1(10)$ \\
\hline Schistosomiasis & $2(20)$ \\
\hline $\mathrm{PBC}$ & $1(10)$ \\
\hline Exposed to antibacterial agents, $n$ (\%) & $8(80)$ \\
\hline SFP, $n(\%)$ & $6(60)$ \\
\hline \multicolumn{2}{|l|}{ ACLF diagnosis when paracentesis, $n(\%)$} \\
\hline Non-ACLF & $7(70)$ \\
\hline ACLF-1 & $2(20)$ \\
\hline ACLF-2 & $1(10)$ \\
\hline Child-Pugh score, mean (SD) & $11(2)$ \\
\hline MELD score, mean (SD) & $22(8)$ \\
\hline SIRS, $n(\%)$ & $2(20)$ \\
\hline Ascites WBC count, median (IQR) & $751(104-8740)$ \\
\hline Ascites PMN count, median (IQR) & $400(66-7208)$ \\
\hline \multicolumn{2}{|l|}{ Type of strain isolated, $n(\%)$} \\
\hline Candida albicans & $8(80)$ \\
\hline Candida glabrata & $1(10)$ \\
\hline Triochosporon & $1(10)$ \\
\hline
\end{tabular}

$H B V$ hepatitis B virus, $P B C$ primary biliary cirrhosis, $S F P$ spontaneous fungi peritonitis, $A C L F$ acute-on chronic liver failure, $M E L D$ model for end-stage liver disease, SIRS systemic inflammatory response syndrome, $W B C$ white blood cell, $P M N$ absolute polymorphonuclear leukocyte

clinical characteristics and outcomes of fungiascites and SFP are shown in Table 2.

\section{Antifungal Treatment}

The drug sensitivity results were not available in four patients since the test was not performed. Meanwhile, results of the remaining six patients showed that the fungus was susceptible to antifungal drugs (5-fluorocytosine, amphotericin B, fluconazole, itraconazole, and voriconazole) (Table 3$)$. Three $(30 \%)$ of the patients with spontaneous fungal infections did not receive antifungal treatment during hospitalisation because they died undiagnosed because of delays in the reporting of laboratory results (Table 3). Eight of the ten patients received empirical antibiotic treatment prior to the administration of antifungal treatment. Of 
Table 2 Clinical characteristics between SFP and fungiascites groups

\begin{tabular}{|c|c|c|}
\hline & Fungiascites $(N=4)$ & $\operatorname{SFP}(N=6)$ \\
\hline Age (years), mean (SD) & $65(18)$ & $63(10)$ \\
\hline Male, $n(\%)$ & $4(100)$ & $3(50)$ \\
\hline \multicolumn{3}{|l|}{ Clinical symptoms, $n$ (\%) } \\
\hline Abdominal pain & $2(50)$ & $1(16.7)$ \\
\hline Fever & - & $3(50.0)$ \\
\hline Insensitive to diuretics & $2(50)$ & $2(33.3)$ \\
\hline New-onset/worsening HE & - & $1(16.7)$ \\
\hline \multicolumn{3}{|l|}{ Concomitant infection, $n(\%)$} \\
\hline Pneumonia, $n$ (\%) & $1(25)$ & $2(33.3)$ \\
\hline Bacteraemia (positive blood culture), $n$ (\%) & - & $1(16.7)$ \\
\hline Urinary tract infection, $n(\%)$ & - & - \\
\hline Skin and soft tissue infection, $n(\%)$ & - & - \\
\hline Ascites white blood cell $\left(\times 10^{6} / \mathrm{L}\right)$, median (IQR) & $88(16-406)$ & $7420(855-10,129)$ \\
\hline Ascites neutrophil $\left(\times 10^{6} / \mathrm{L}\right)$, median (IQR) & $12(2-120)$ & $4642(382-9489)$ \\
\hline Ascites total protein $(\mathrm{g} / \mathrm{L})$, mean $(\mathrm{SD})$ & $19.9(6.5)$ & $22.6(7.2)$ \\
\hline \multicolumn{3}{|l|}{ Laboratory measurements } \\
\hline Bilirubin $(\mu \mathrm{mol} / \mathrm{L})$, median $(\mathrm{IQR})$ & $121.5(53.3-408.5)$ & $41.0(29.1-151.7)$ \\
\hline INR, median (IQR) & $1.8(1.3-2.2)$ & $1.5(1.3-1.8)$ \\
\hline Serum creatinine $(\mu \mathrm{mol} / \mathrm{L})$, median $(\mathrm{IQR})$ & $153.9(96.2-366.3)$ & $116.3(63.5-355.5)$ \\
\hline Leukocytes $\left(\times 10^{9} / \mathrm{L}\right)$, median (IQR) & $10.9(7.5-16.9)$ & $9.9(5.3-18.7)$ \\
\hline C-reactive protein $(\mathrm{mg} / \mathrm{L})$, median (IQR) & $43.4(26.7-60.0)$ & $76.7(66.9-119.3)$ \\
\hline Serum sodium $(\mathrm{mmol} / \mathrm{L})$, mean $(\mathrm{SD})$ & $122.7(20.1)$ & $133.1(8.5)$ \\
\hline Albumin $(\mathrm{g} / \mathrm{L})$ & $25.7(5.2)$ & $26.5(7.1)$ \\
\hline ACLF, $n(\%)$ & $1(25)$ & $2(33.3)$ \\
\hline AKI, $n(\%)$ & $3(75)$ & $3(50)$ \\
\hline Sepsis diagnosis, $n$ (\%) & $1(25)$ & $1(16.7)$ \\
\hline Child-Pugh score, mean (SD) & $12(1)$ & $10(2)$ \\
\hline MELD score, mean (SD) & $26(7)$ & $20(9)$ \\
\hline Hospital stay (days), median (IQR) & $13(3-23)$ & $19(10-56)$ \\
\hline 28-day mortality, $n$ (\%) & $3(75)$ & $3(50)$ \\
\hline
\end{tabular}

$\overline{S F P}$ spontaneous fungal peritonitis, $A C L F$ acute-on-chronic liver failure, $A K I$ acute kidney injury, $H E$ hepatic encephalopathy, $M E L D$ model for end-stage liver disease, $I Q R$ interquartile range 


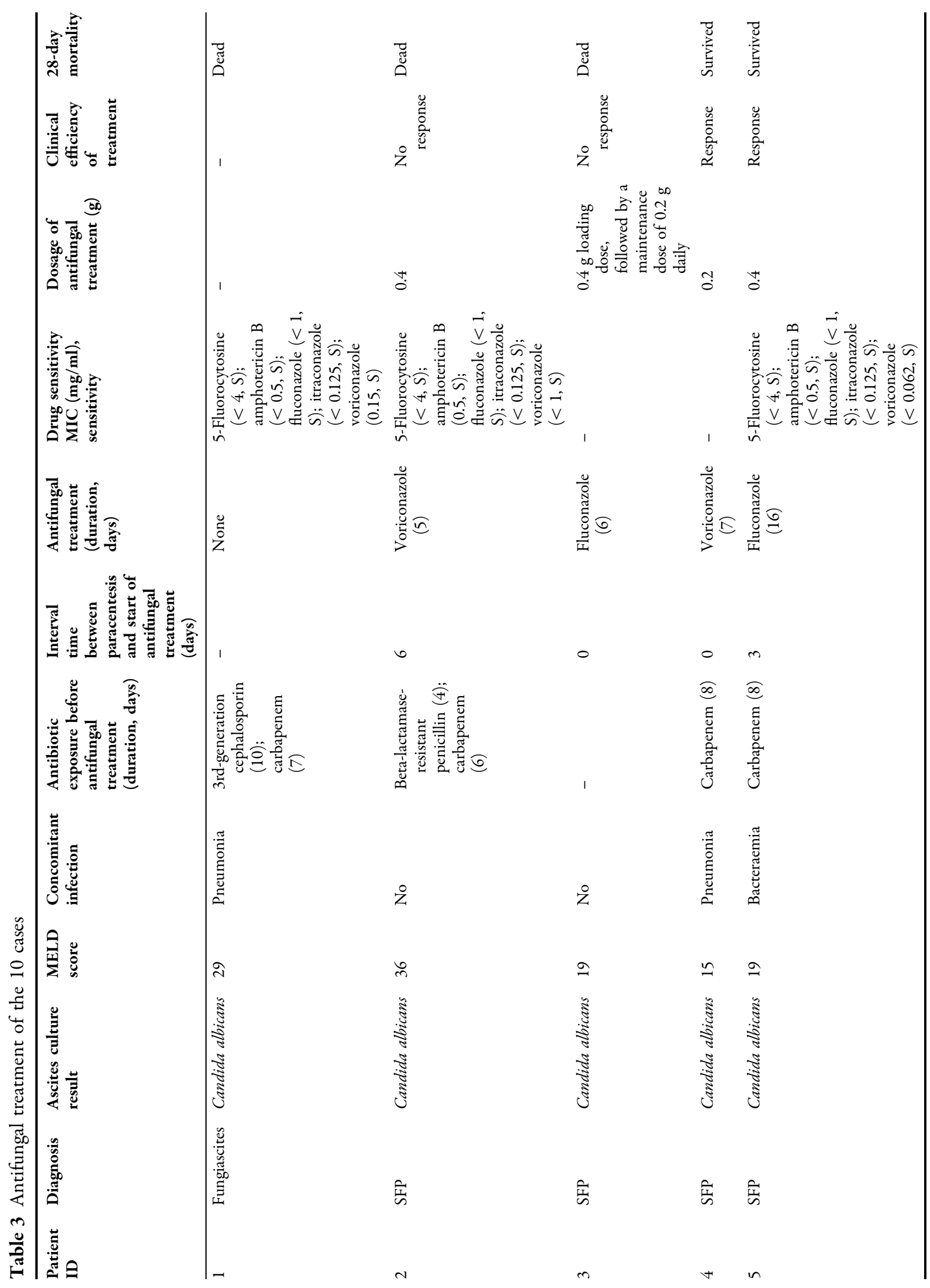




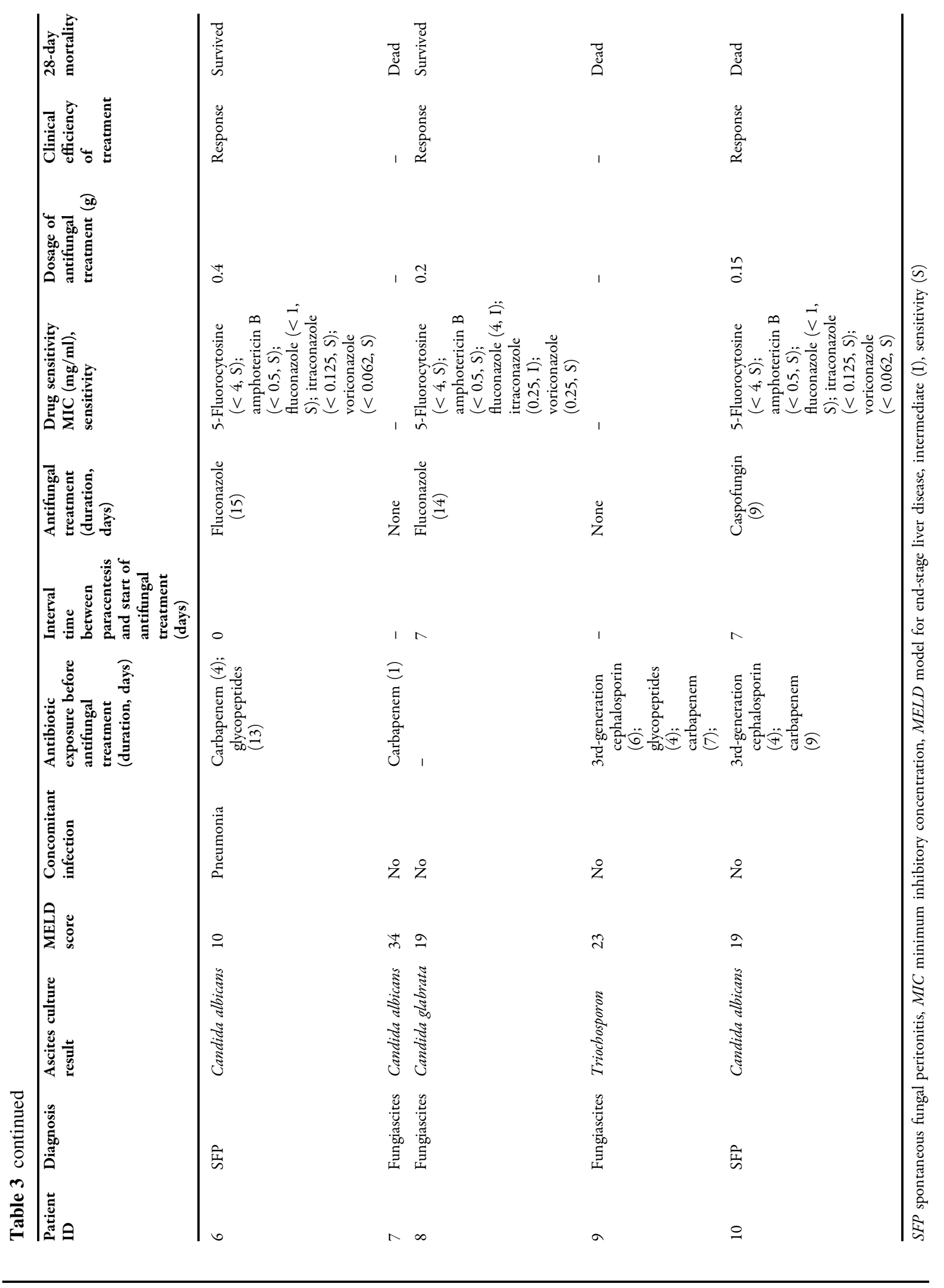


the patients treated with antifungal agents, three received empirical antifungal treatment on the day of the paracentesis procedure. The remaining four patients received antifungal treatment after the ascites culture result was returned. The most frequent antifungal agent used was fluconazole, followed by voriconazole. The dosages of antifungal agents administered to patients are presented in Table 3. Approximately, $71.4 \%$ (5 of 7 ) of the patients responded to antifungal therapy, and four of these patients survived through 28 days. The median duration of antifungal treatment was 9 days (range 5-16 days).

\section{DISCUSSION}

We retrospectively described the spontaneous fungal ascites infection that developed in ten patients with liver cirrhosis, and Candida albicans was the most frequent aetiologic agent. Patients with cirrhosis and spontaneous fungal infection had non-negligible AKI and 28-day mortality rates, which may be related to the delayed diagnosis of ascites fungal infection.

SBP is a common complication in patients with cirrhosis and ascites. A previous study reported that the incidence of SBP ranged from $10 \%$ to $30 \%$ [17]. Currently, multidrug-resistant bacterial infection in patients with end-stage liver disease has become a global threat, leading to empirical antibiotic treatment failure [16]. However, spontaneous fungal ascites infection rarely occurs, with a rate of less than $5 \%$, but causes severe complications in patients with cirrhosis. Recent descriptions of disturbed intestinal fungome argue that translocation of these microorganisms may be associated with infection and progression as is seen with bacterial translocation in patients with cirrhosis $[18,19]$. This study showed that patients with spontaneous fungal ascites infection had a high incidence of AKI and 28-day mortality rate, which is in line with the results of a previous study [20]. In this study, three of four fungiascites patients $(75 \%)$ did not receive any antifungal treatment, indicating that the potential impact of fungal infection may be underestimated in routine clinical setting, since these patients had poor outcomes. In the clinical practice, the indication for initiating empirical antimicrobial treatment is ascites neutrophil greater than 250 cells $/ \mathrm{mm}^{3}$. Nerveless, current guidelines do not indicate the recommended treatment for fungiascites [4]. Hence, this study suggests that the threshold of ascites neutrophil counts to initiate antifungal treatment should be low, particularly in those with severely advanced liver disease.

Patients with critical liver disorders have higher risk of developing infection, including fungal infection. In current clinical practice, liver transplantation is the only treatment method for end-stage liver disease. Active fungal infection is a contraindication for transplantation. For patients with SBP, the impatient mortality decreased over past decades (19.1\% to $16.1 \%$ ) because of the effective use of empirical antibiotics treatment. Our results show that spontaneous fungal ascites infection that occurred in patients with cirrhosis had nonnegligible complications, especially AKI and progression to ACLF. We speculated that this may attribute to the improper use of empirical antibiotics which targeted bacteria and the delay diagnosis of fungal infection. Hence, early diagnosis of fungal infection is urgently needed. However, the diagnosis of spontaneous fungal ascites infection depends on the results of traditional ascites culture and usually takes a few days (3-5 days), thus leading to delays in the initiation of therapy in patients with cirrhosis. Recently, metagenomics next-generation sequencing (mNGS) has proved to be a promising approach for the diagnosis of infectious diseases covering a wide range of pathogens, including bacteria, fungi, and viruses [21], and the results of mNGS can be obtained within $48 \mathrm{~h}$. Thus, early identification of high-risk patients who are prone to fungal infection and early diagnosis are the key steps in improving the patient's clinical outcomes. Conducting ascites mNGS in patients with cirrhosis may lead to their timely treatment.

With regard to the treatment of spontaneous fungal ascites infection, neither EASL nor Chinese guidelines provide recommendations on the management of ascites in patients with cirrhosis [4, 22]. Different antifungal agents 
were used in this study: voriconazole, fluconazole, and caspofungin. The differences in the antifungal regimen may relate to the severity of liver and renal function and the clinical experience. A previous study showed that ascites infections are the most common triggering factors of AKI and strongly affect the outcome of patients with cirrhosis and ascites [23]. In this study, AKI was more prevalent in patients who developed spontaneous fungal ascites infection. For patients with SBP, current guidelines recommend that the transfusion of human serum albumin (HSA) significantly reduces the incidence of AKI [24]. However, the dosage of HSA for patients with SFP and fungiascites remains unclear. Therefore, the treatment of SFP and fungiascites, including antifungal agents and dosage of HSA, needs to be explored further in future studies.

This study has several limitations. First, it is a retrospective study, which possibly includes information bias. Second, although our study reported high AKI incidence and 28-day mortality rate, the difference in these outcomes between patients with SFP and those with fungiascites could not be clearly demonstrated because of the small sample size. Hence, related studies with a larger sample size should be conducted to further evaluate the outcomes of patients with fungal ascites infection. Finally, the microbiological findings may be representative of primary or secondary fungal infections since the blood cultures were not performed in all patients with cirrhosis.

\section{CONCLUSION}

Patients with cirrhosis and spontaneous fungal ascites infection had poor outcomes. Hence, fungal cultures of ascitic fluid from patients with cirrhosis should be performed to ensure optimal clinical management, especially from those with critical liver disease and poorly administered empirical antimicrobial therapy. Future studies should focus on the early diagnosis of fungal infection and optimise antifungal treatment in patients with cirrhosis.

\section{ACKNOWLEDGEMENTS}

Funding. This work was supported by grants from National Science and Technology Major Project (2018ZX10723203, 2018ZX10302206); National Key Research and Development Program of China (2017YFC0908100); Local Innovative and Research Teams Project of Guangdong Pearl River Talents Program (2017BT01S131); Clinical Research Program of Nanfang Hospital, Southern Medical University (2018CR037, 2020CR022); Clinical Research Startup Program of Southern Medical University by High-level University Construction Funding of Guangdong Provincial Department of Education (LC2019ZD006, LC2016PY005) and President Foundation of Nanfang Hospital, Southern Medical University (2019Z003). Department of Science and Technology of Guangdong Province (2014B020228003, 2015B020226004). The Rapid Service Fees were funded by the authors.

Authorship. All named authors meet the International Committee of Medical Journal Editors (ICMJE) criteria for authorship for this article, take responsibility for the integrity of the work as a whole, and have given their approval for this version to be published.

Authorship Contributions. BL and CY: study concept and design, acquisition of data, analysis and interpretation of data, drafting of the manuscript. XW, ZQ, YH, GZ: acquisition of data, analysis and interpretation of data. JC: study concept and design, drafting of the manuscript, critical revision of the manuscript for important intellectual content, approval of the final version of the manuscript, statistical analysis, study supervision, obtained funding.

Disclosures. Beiling Li, Chao Yang, Xianbo Wang, Zhiping Qian, Yan Huang, Guotao Zhong and Jinjun Chen declare no conflict of interest.

Compliance with Ethics Guidelines. The study received ethics approval from the ethics committee of all the participating medical 
centers (Nanfang Hospital, Shanghai Public Health Clinical Centre, Xiangya Hospital and Beijing Ditan Hospital). The ethics committees waived the informed consent because the data were de-identified and individual patient's data were not published. The study was performed in accordance with the declaration of Helsinki 1964 and its later amendments.

Data Availability. The datasets generated during and/or analysed during the current study are available from the corresponding author on reasonable request.

Open Access. This article is licensed under a Creative Commons Attribution-NonCommercial 4.0 International License, which permits any non-commercial use, sharing, adaptation, distribution and reproduction in any medium or format, as long as you give appropriate credit to the original author(s) and the source, provide a link to the Creative Commons licence, and indicate if changes were made. The images or other third party material in this article are included in the article's Creative Commons licence, unless indicated otherwise in a credit line to the material. If material is not included in the article's Creative Commons licence and your intended use is not permitted by statutory regulation or exceeds the permitted use, you will need to obtain permission directly from the copyright holder. To view a copy of this licence, visit http://creativecommons.org/licenses/by$\mathrm{nc} / 4.0 /$.

\section{REFERENCES}

1. Arvaniti V, D'Amico G, Fede G, et al. Infections in patients with cirrhosis increase mortality four-fold and should be used in determining prognosis. Gastroenterology. 2010;139(1246-1256):1251-6.

2. Runyon BA. Management of adult patients with ascites due to cirrhosis. Hepatology. 2004;39: 841-56.

3. European Association for the Study of the Liver. EASL clinical practice guidelines on the management of ascites, spontaneous bacterial peritonitis, and hepatorenal syndrome in cirrhosis. J Hepatol. 2010;53:397-417.
4. European Association for the Study of the Liver. EASL Clinical Practice Guidelines for the management of patients with decompensated cirrhosis. J Hepatol. 2018;69:406-60.

5. Koulenti D, Garnacho-Montero J, Blot S. Approach to invasive pulmonary aspergillosis in critically ill patients. Curr Opin Infect Dis. 2014;27:174-83.

6. Bienvenu AL, Walter T, Boulez F, et al. Acute liver failure may lead to lethal pulmonary aspergillosis. J Clin Gastroenterol. 2010;44:593-4.

7. Wu Z, Ling Z, Shao F, Sheng J, Li L. Invasive pulmonary aspergillosis in patients with acute-onchronic liver failure. J Int Med Res. 2012;40: 1958-65.

8. Chen J, Yang Q, Huang J, Li L. Risk factors for invasive pulmonary aspergillosis and hospital mortality in acute-on-chronic liver failure patients: a retrospective-cohort study. Int J Med Sci. 2013;10: $1625-31$.

9. Gustot $\mathrm{T}$, Maillart $\mathrm{E}$, Bocci $\mathrm{M}$, et al. Invasive aspergillosis in patients with severe alcoholic hepatitis. J Hepatol. 2014;60:267-74.

10. Gao J, Zhang Q, Wu Y, et al. Improving survival of acute-on-chronic liver failure patients complicated with invasive pulmonary aspergillosis. Sci Rep. 2018;8:876.

11. Albillos A, Lario M, Alvarez-Mon M. Cirrhosis-associated immune dysfunction: distinctive features and clinical relevance. J Hepatol. 2014;61:1385-96.

12. Bucsics T, Schwabl P, Mandorfer M, Peck-Radosavljevic M. Prognosis of cirrhotic patients with fungiascites and spontaneous fungal peritonitis (SFP). J Hepatol. 2016;64:1452-4.

13. Jones RN, Glick T, Sader HS, et al. Educational antimicrobial susceptibility testing as a critical component of microbiology laboratory proficiency programs: American Proficiency Institute results for 2007-2011. Diagn Microbiol Infect Dis. 2013;75: 357-60.

14. Angeli P, Gines P, Wong F, et al. Diagnosis and management of acute kidney injury in patients with cirrhosis: revised consensus recommendations of the International Club of Ascites. J Hepatol. 2015;62:968-74.

15. Moreau R, Jalan R, Gines P, et al. Acute-on-chronic liver failure is a distinct syndrome that develops in patients with acute decompensation of cirrhosis. Gastroenterology. 2013;144(1426-1437):1431-7.

16. Piano S, Singh V, Caraceni P, et al. Epidemiology and effects of bacterial infections in patients with 
cirrhosis worldwide. Gastroenterology. 2019;156: 1368-80.

17. Dever JB, Sheikh MY. Review article: spontaneous bacterial peritonitis-bacteriology, diagnosis, treatment, risk factors and prevention. Aliment Pharmacol Ther. 2015;41:1116-31.

18. Bajaj JS, Khoruts A. Microbiota changes and intestinal microbiota transplantation in liver diseases and cirrhosis. J Hepatol. 2020;72:1003-27.

19. Jiang L, Starkel P, Fan JG, et al. The gut mycobiome: a novel player in chronic liver diseases. J Gastroenterol. 2021;56:1-11.

20. Huang $\mathrm{CH}$, Pang LT, $\mathrm{Xu}$ LC, et al. Risk factors, clinical features, and short-term prognosis of spontaneous fungal peritonitis in cirrhosis: a matched case-control study. World J Clin Cases. 2019;7:2438-49.

21. Forbes JD, Knox NC, Ronholm J, Pagotto F, Reimer A. Metagenomics: the next culture-independent game changer. Front Microbiol. 2017;8:1069.

22. Xu X, Duan Z, Ding H, et al. Chinese guidelines on the management of ascites and its related complications in cirrhosis. Hepatol Int. 2019;13:1-21.

23. Llovet JM, Bru C, Bruix J. Prognosis of hepatocellular carcinoma: the BCLC staging classification. Semin Liver Dis. 1999;19:329-38.

24. Sort P, Navasa M, Arroyo V, et al. Effect of intravenous albumin on renal impairment and mortality in patients with cirrhosis and spontaneous bacterial peritonitis. N Engl J Med. 1999;341:403-9. 